\title{
Elastic properties of self-organized nanogratings produced by femtosecond laser exposure of fused silica
}

\author{
Pieter Vlugter (1) and Yves Bellouard (1) \\ Galatea Lab, STI/IMT, Ecole Polytechnique Fédérale de Lausanne (EPFL), 2002 Neuchâtel, Switzerland
}

(Received 3 November 2019; accepted 31 January 2020; published 27 February 2020)

\begin{abstract}
Under certain exposure conditions, a femtosecond laser beam focused in the bulk of fused silica leads to the formation of self-organized structures consisting of a series of "nanolayers," parallel to one another. Remarkably, this laser-induced nanoscale anisotropy offers the possibility to locally engineer macroscopic properties of a given substrate by selectively exposing it in arbitrarily chosen locations to a laser beam with designed polarization states. Although various physical properties are affected by the laser, this paper specifically discusses in-plane elastic properties of these nanostructures. Using a method based on monitoring resonant properties of vibrating cantilevers combined with a mechanical model of the nanostructures, the Young's moduli of individual nanolayers are calculated and used to define the stiffness matrix of the composite structure. The model shows a good agreement with measured mechanical properties of arbitrarily oriented nanostructures. This work demonstrates the predictability and controllability of laser-induced nanoscale mechanical properties and offers a framework for engineering arbitrary elastic properties through 3D laser writing.
\end{abstract}

DOI: 10.1103/PhysRevMaterials.4.023607

\section{INTRODUCTION}

Femtosecond laser bulk exposure can lead to the formation of self-organized nanolayers parallel to one another that are commonly referred to as nanogratings [1]. Since their discovery at the beginning of the 2000 s, their formation and properties have been extensively studied [2-4]. These nanogratings are characterized by a self-organized modulation of nanoporous layers [5], roughly spaced by half the wavelength $(\lambda / 2 n)[6]$ and perpendicularly aligned with the laser beam electrostatic field that defines its polarization state [2]. In fused silica, these structures are typically found for pulse durations and pulse energies above $\sim 200 \mathrm{fs}$ and $~ 200 \mathrm{~nJ}$ [7], respectively. Postmortem experimental studies show a clear evolution of the self-organization with the increasing number of pulses [8,9], with a temporal pulse separation far longer than the lifetime of the induced plasma and self-trapped excitons [10], suggesting that the premodified material triggers the self-organization process [11]. The exact mechanism leading to the creation of these nanofeatures [2,10-13] is to date not established. Nonetheless, this type of modification has been used in various photonics applications, such as polarization converters [14] or optical memory devices [15], but also for controlling the stress state in materials [16,17], inducing controlled displacements [18-20], or for tuning properties, such as the coefficient of thermal expansion [21]. To date, these changes of thermomechanical behavior cannot be fully understood, predicted, and controlled without a deeper

\footnotetext{
*pieter.vlugter@epfl.ch
}

Published by the American Physical Society under the terms of the Creative Commons Attribution 4.0 International license. Further distribution of this work must maintain attribution to the author(s) and the published article's title, journal citation, and DOI. understanding of the mechanical properties of the nanogratings. To fill this gap of knowledge, here we report on the estimation of the nanolayers Young's moduli using a resonant cantilever-based experiment combined with a mechanical model of the nanostructure. From an application point of view, this precise knowledge of the mechanical properties is essential for thermal expansion compensating schemes and thermomechanical devices, as well for stress-induced functionalities, like, for instance, used in photonics devices $[16,17]$, mechanical positioners $[19,20]$, and for tuning the resonance frequency of mechanical resonators.

\section{EXPERIMENTAL PROCEDURE}

The resonance frequency of a mechanical structure depends on its mass and its stiffness $(f \sim \sqrt{k / m})$. Therefore, by modifying the constitutive material of a vibrating structure using laser irradiation a noticeable frequency shift of its resonance is expected, as a change in Young's modulus and mass distribution occurs Ref. [18,28]. Here, we use this principle to evaluate the local change of Young's modulus. Our resonating element consists of a free-end cantilever, shown in Fig. 1(a), where the red-colored zone illustrates the lasermodified volume. Considering a maximum volume expansion of the exposed volume of $0.03 \%$ [4], we estimate that the frequency change due to mass redistribution is negligible. The lowest resonant frequency of a clamped-free cantilever is expressed in Eq. (1). $\rho$ and $V$ are the mass density and the volume of the cantilever. $E$ and $I$ are the Young's modulus and the second moment of inertia, respectively, with the subscripts $l$ and $\mathrm{a}_{s}$ referring to the laser-affected zones (LAZs) and the nonexposed ones, respectively. In Fig. 1(b), a cross-section view $(y-z$ plane) of the cantilever is given, with the different zones indicated. $E_{l, t}$ is expressed in terms of the volume fractions, $V_{l}$ and $V_{s}$, the two Young's moduli $E_{l, t}$ or $E_{l, a}$ (i.e., the transverse and the axial moduli), and $E_{s}$ retrieved 
(a)
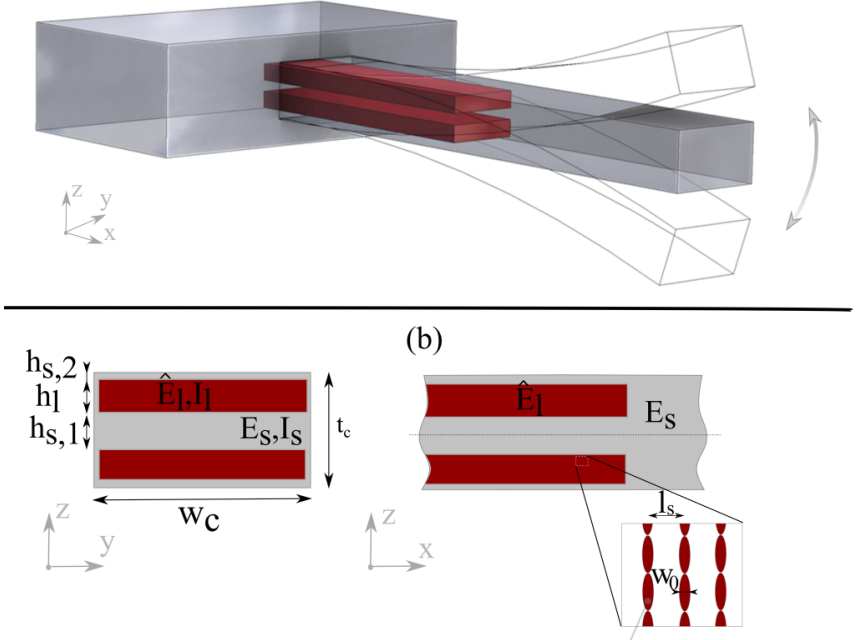

\begin{tabular}{cc} 
Dimension & Value $(\mu \mathrm{m})$ \\
\hline$t_{c}$ & 333 \\
$l_{c}$ & 6000 \\
$w_{c}$ & 750 \\
$h_{s 1}$ & 110 \\
$h_{s 2}$ & 20 \\
$h_{l}$ & 90 \\
$l_{s}$ & 4 \\
$w_{0}$ & 1.5
\end{tabular}

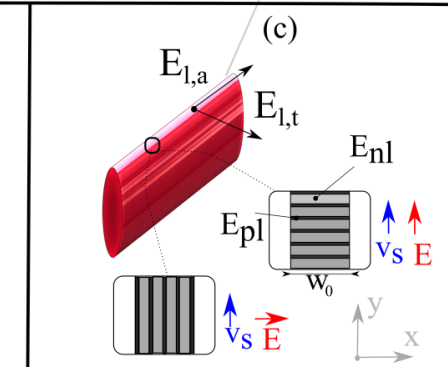

FIG. 1. (a) Schematic of the test device: A cantilever (in gray) is locally exposed by the laser in its upper and lower volumes (shown in red). (b) Front and side cross-sectional views of the laser exposed volumes with the relevant dimensions indicated in the table at the bottom left. (c) A laser-affected zone (LAZ) with the transverse and axial Young's modulus indicated, $E_{l, t}$ and $E_{l, a}$, and in the magnified views, the orientation of the nanogratings detailed for two polarization cases, $0^{\circ}$ and $90^{\circ}$ defined with respect to the writing direction.

from Eq. (1). When the lines are written perpendicular to the cantilever, as defined in Fig. 1(c), Eq. 2(a) is used for obtaining the expression of $\hat{E}_{l}$, while Eq. 2(b) is used when lines are written along the cantilever. Let us for now only consider the transverse properties [and, therefore, Eq. 2(a)]. The volume fractions are defined in this case as $V_{l}=w_{0} / l_{s}$ and $V_{s}=1-V_{l}$ :

$$
\begin{gathered}
f=\frac{1.875^{2}}{2 \pi l_{c}^{2}} \sqrt{\frac{\hat{E}_{l} I_{l}+E_{s} I_{s}}{\rho A}} \quad \text { with } \quad \hat{E}_{l}=\frac{E_{l, t} E_{s}}{E_{l, t} V_{s}+E_{s} V_{l}}, \\
\mathrm{a}: \hat{E}_{l}=\frac{E_{l, t} E_{s}}{E_{l, t} V_{s}+E_{s} V_{l}} \quad \mathrm{~b}: \quad \hat{E}_{l}=E_{l, a} V_{l}+E_{s} V_{s} .
\end{gathered}
$$

The resonator is fabricated using femtosecond laser exposure and a subsequent chemical etching step according to the procedure in Ref. [22], and further exposed using the same femtosecond laser, an Yb-doped fiber amplified system (from amplitude, emitting 380 fs pulses at a wavelength of $1030 \mathrm{~nm})$. The roughness of the cantilevers is around $250 \mathrm{~nm}(\mathrm{Ra})$, as characterized in Ref. [23]. A piezoelectric transducer excites the resonator and a contactless displacement sensor (Keyence LKH022) measures the tip displacements. To track down and maintain the system at its resonance

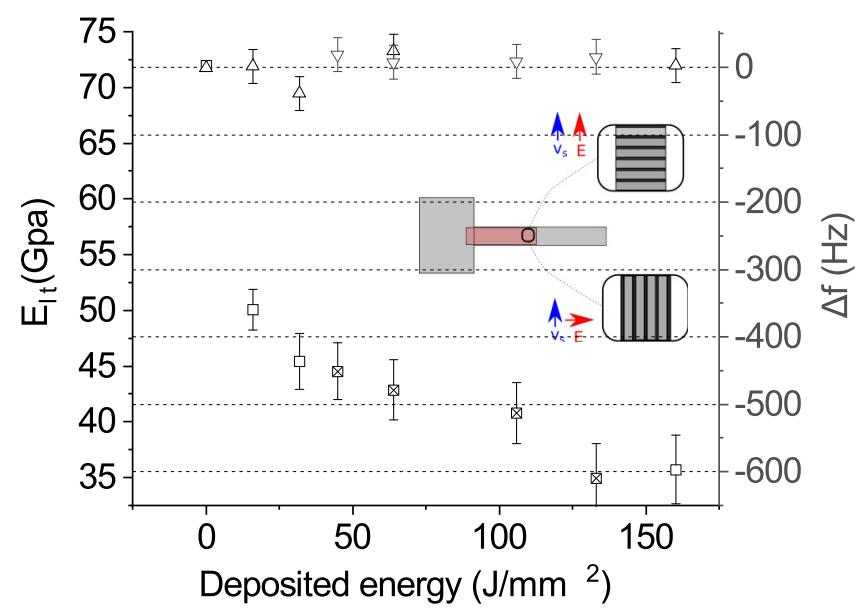

FIG. 2. Measured frequency shifts and estimated $E_{l, t}$ as function of deposited energy for two polarization cases. The triangles and squares represent the $0^{\circ}$ and $90^{\circ}$ polarizations, respectively, and for which the angles are defined relatively to the writing direction.

frequency, a phase-lock loop method is applied using a lock-in amplifier (Stanford Research Systems SR850) and a proportional-integral controller. The resonance ensemble is placed in an environmental chamber, i.e., a well-controlled temperature (within $\pm 0.1 \mathrm{~K}$ ), nitrogen-rich environment, and controlled flow conditions, to minimize external disturbances. In these experiments, 1-mm-thick fused silica (Corning 7980 OF) substrates were used, with each of them containing five resonators. On each substrate, one resonator is left unexposed and is used as a reference. The four others are exposed with a fixed pulse energy of $240 \mathrm{~nJ}$ and a repetition rate of $750 \mathrm{kHz}$.

\section{Measurement principle and estimation of nanolayers Young's moduli}

To be able to discriminate between the two Young's moduli of nanolayers defining the nanogratings, two sets of exposed cantilevers are used, with orthogonal polarization states. Using a composite expression for the effective Young's modulus of the ensemble, one can formulate two independent equations, out of which are the actual values of the Young's modulus for the nanolayers. In practice, two nanograting orientations, oriented along and perpendicular with respect to the cantilever principal axis, are selected for solving the system of equations. The deposited energy is changed by varying the translation speed from 1 to $10 \mathrm{~mm} / \mathrm{s}$ - which corresponds to a pulse number range $75-750[-/ \mu \mathrm{m}]$. Finally, for these experiments, the lines were written perpendicular to the cantilever, as depicted in Fig. 1(c). Figure 2 shows the measured frequency shift and the corresponding estimate of the Young's modulus for the transverse direction, using Eq. (1), for the two polarization cases, with respect to the reference resonator and as a function of the deposited energy. The data points on zero deposited energy represent the reference resonator. For a zero-degree polarization, nanogratings are oriented transverse to the writing direction, a slight positive frequency shift is observed revealing a stiffer material than the pristine material. For a polarization of $90^{\circ}$, i.e., nanogratings orientated transverse to the writing direction, large negative values of the resonance frequency shift are observed. For this case, $E_{l, t}$ 


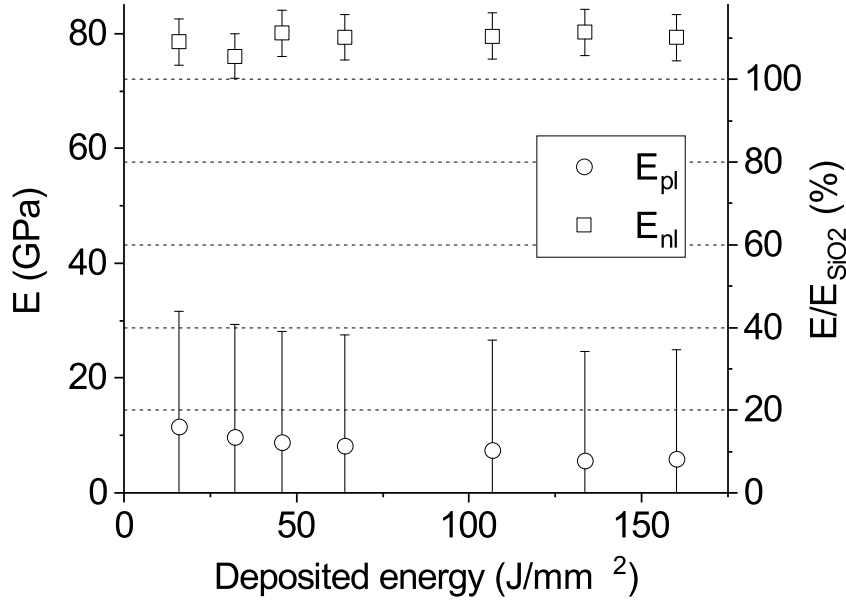

FIG. 3. Estimated local properties of porous $\left(E_{\mathrm{pl}}-0\right)$ and nonporous layers $\left(E_{\mathrm{nl}}-\square\right)$. The Young's modulus of the pristine material is $72 \mathrm{GPa}$.

decays from $50 \mathrm{GPa}$ to $35 \mathrm{GPa}$, going from lower to higher deposited energies. Athanasiou and Bellouard [18] made an estimate, by indirect means of the transverse Young's modulus with similar exposure conditions-a polarization of $90^{\circ}$, pulse energy $\sim 210 \mathrm{~nJ}$, and deposited energy $\sim 20 \mathrm{~J} / \mathrm{mm}^{2}$. They report a significant decrease of the elastic constant, with an estimated value of $\sim 30 \mathrm{GPa}$. At this stage, it is not clear if the difference of about $10 \mathrm{GPa}$ with our observation is due to the difference in exposure conditions or to the measurement method. Nevertheless, both methods show a similar trend.

For a polarization aligned along and perpendicular to the writing direction, the nanogratings are parallel and serially arranged, with respect to the cantilever long axis. Based on the results reported by Ref. [24] and obtained using similar laser exposure conditions, we estimate the nanograting periodicity variation to be less than $5 \%$ for the pulse overlapping densities considered here. For the two nanolayer arrangements, we can express $E_{l, t}$ in terms of volume fractions and local Young's moduli. This is outlined in Eqs. (3) and (4), where $E_{\mathrm{pl}}$ and $E_{\mathrm{nl}}$ are the Young's modulus of the porous layer of the surrounding layer, respectively. $V_{\mathrm{nl}}$ and $V_{\mathrm{pl}}$ are the corresponding volume fractions. $V_{\mathrm{pl}}$ defines the ratio between the width of porous nanolayers and the spatial modulation period of nanolayers. Based on observations from others [9,25], we chose $V_{\mathrm{pl}}=0.1$ as the value. By definition, the volume fraction of the intermediate layers is expressed by $V_{\mathrm{nl}}=1-V_{\mathrm{pl}}$.

$$
\begin{gathered}
E_{l, t}=E_{\mathrm{nl}} V_{\mathrm{nl}}+E_{\mathrm{pl}} V_{\mathrm{pl}} \text { for } 0^{\circ} \text { polarization, } \\
E_{l, t}=\frac{E_{\mathrm{pl}} E_{\mathrm{nl}}}{E_{\mathrm{nl}} V_{\mathrm{pl}}+E_{\mathrm{pl}} V_{\mathrm{nl}}} \text { for } 90^{\circ} \text { polarization. }
\end{gathered}
$$

The estimated values of the local elastic properties are depicted in Fig. 3. It is found that the porous layers $\left(E_{\mathrm{nl}}\right)$ have values of Young's modulus $\sim 90 \%$ lower compared to the host material $\left(\mathrm{SiO}_{2}\right)$ and that the intermediate layers $\left(E_{\mathrm{pl}}\right)$ (in between porous layers) stiffen, eventually reaching a mean value of $80 \mathrm{GPa}$.

The decrease in Young's modulus for the porous layer can be understood as the lowering of the material density

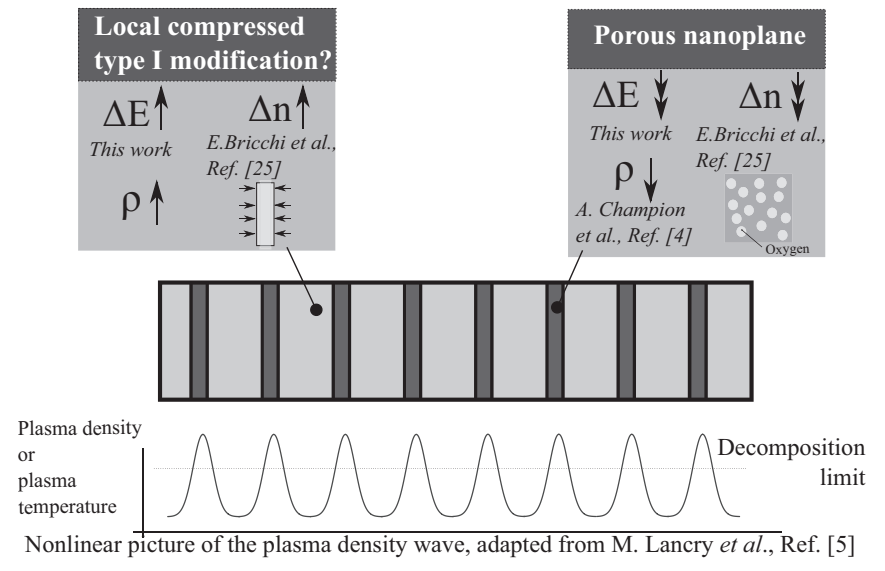

FIG. 4. An illustrative summary of the discussion to explain our observations, within the middle of an illustration of the nanograting structure. Top right: An illustration of the predicted material properties and chemical composition signature of a porous layer-the refractive index change estimated by Ref. [25], the oxygen-filled pores [5], and elastic modulus estimated in this paper. On the top left, a refractive index increase [25] and the elastic modulus increase. Below the spatial distribution of plasma density, with peaks exceeding the decomposition limit, adapted from Ref. [5].

as the addition of pores effectively limits the number of interatomic bonds that can store elastic energy when being strained [26,27]. The presence of free molecular oxygens, found by Raman and fluorescent spectroscopy [5,28,29], supports a decrease in the matrix connectivity. The increase of the Young's modulus $\left(E_{\mathrm{pl}}\right)$ is an interesting observation and is, to date, not directly supported by other types of experimental observations, due to the lack of a suitable technique having the resolution to effectively measure only the interstitial layer. Note that for a so-called type-I modification, a Young's modulus increase of about 2 to $3 \mathrm{GPa}$ was measured using nanoindentation [30]. Here, the observed stiffening effect implies that this zone gets denser. Furthermore, a densification of these nonporous layers is also indirectly suggested in Ref. [31], where they estimate an increase of the refractive index of this layer by analyzing the strong form birefringence induced by the nanogratings. The increase of the Young's modulus, refractive index, and possible densification is like a regime-I modification [28,30,32] which exhibited similar characteristics. Previous works [5,29] made similar observations. Lancry et al. [5] proposed a plasma density profile where, in the nonporous layers, the density meets the conditions for a regime-I modification, while in the position of the porous nanolayers, a decomposition threshold for $\mathrm{SiO}_{2}$ molecules is exceeded. In this paper, the measured values of the local Young's modulus increase and its corresponding refractive index increase, from $2 \times 10^{-2}$ to $5 \times 10^{-2}[-]$ as measured in Ref. [25], are significantly higher than the ones found for the regime-I modification [33,34], where an increase from $10^{-4}$ to $10^{-3}[-]$ is reported. The mechanisms which have been accounted for the refractive index increase for laser irritated material are (i) compaction due to the increase of fictive temperature by rapid solidification [35], (ii) increase of density due to bond breaking and reorganization [36], (iii) color center formation [37], or simply (iv) mechanical 
compression [38,39]. The increase of either the Young's modulus or refractive index cannot be explained by the fictive temperature increase only, as it may for a regime-I modification. The studies about fictive temperature increase show a $\Delta E \approx 1.3 \mathrm{GPa}[40]$ and $\Delta n \approx 600 \times 10^{-6}$ [-] [40-42] for a $T_{f}=1600{ }^{\circ} \mathrm{C}$, as no dramatically higher values can be expected as the fictive temperature is limited by the melting temperature. Furthermore, the authors in Refs. [29,36] argue that this thermally driven reorganization is too slow for these ultrashort timescales. As no increase of the Young's modulus is expected due to the formation of color centers-rather a decrease as a result of the lack of connectivity of the $\mathrm{SiO}_{2}$ network-it is more likely that a mechanism based on the occurrence of fast compressive stress combined with thermal quenching is responsible for the high value increases observed in our experiments. In hot-compression studies reported in Ref. [39], an external pressure is applied on heated samples, assisting an accelerating microflow toward a denser structure. In combination with rapid cooling, high densification configurations are quenched and stabilized. While cold-compressed fused silica behaves elastically at pressures below $9 \mathrm{GPa}$ [38], quenched compressed silica shows residual densification and an increase in Young's moduli, with significantly higher thermal stability [39]. A similar effect could occur here, considering the presence of hotspots within the porous layers [5] that pressurize the surrounding material—already at elevated temperatures-due to the intense localized plasma state. Considering our experimental conditions and according to Ref. [43], the temperature decreases after laser exposure, from peak temperatures above $2500{ }^{\circ} \mathrm{C}$ to room temperature, within microseconds. The pressure is maintained after the rapid cooling as free-oxygen molecules are not recombined with the glass matrix and remain trapped in a gas phase within the porous structure permanently as the silica matrix is too dense to be permeable to oxygen. To support our hypothesis, we note that pressures in the order of $2 \mathrm{GPa}$ at elevated temperature are sufficient to reach the values we obtained for the Young's modulus and refractive index [44]. Note that in another work [45], the local stress in a single layer was indirectly predicted to reach about $2 \mathrm{GPa}$, which further supports the present observations. This is in essence contradicting the idea that suggests that the formation of porous layers is not the driving force for compaction, but rather are cracks as a consequence of the neighboring densifying material [10,29]. Figure 4 summarizes illustratively the discussion above.

\section{MODELING OF IN-PLANE ELASTIC PROPERTIES}

In the previous section, we derived the local material properties from experiments. In this section, we use these values to predict in-plane elastic properties as a function of arbitrary nanograting orientations. Specifically, we test the validity of our model for two loading directions, axial and transverse. In

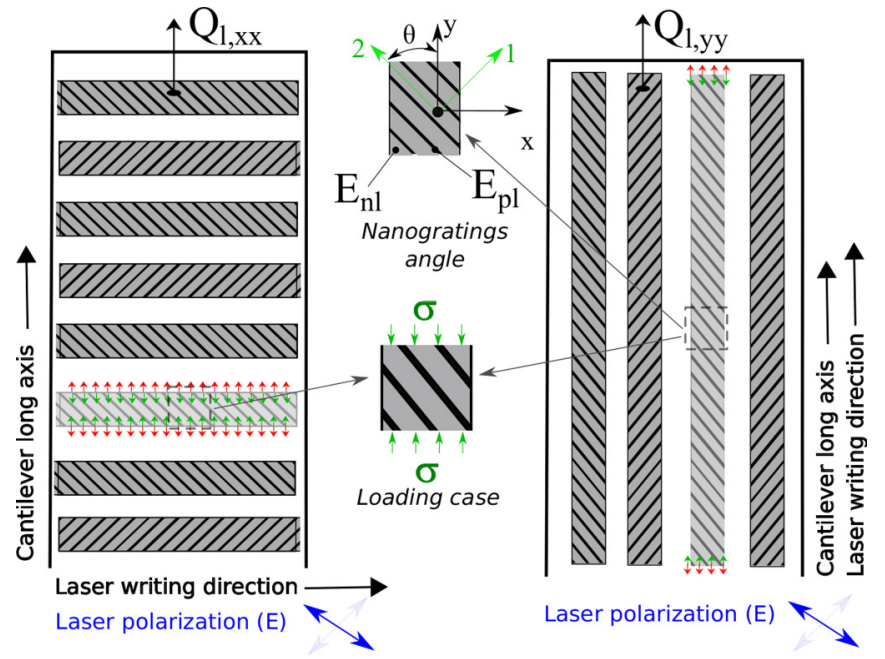

FIG. 5. Schematic of the writing direction strategy, the definition of the nanograting orientation and definition of $Q_{l, x x}$ and $Q_{l, y y}$. On the left, the transverse laser-written lines and at the right longitudinal laser-written lines, with respect to the cantilever long axis. Furthermore, for both cases, the nanogratings are mirrored with respect to the cantilever long axis for each subsequent line. In the middle, the magnified view illustrates nanogratings with a local coordinate frame (1-2) and the global coordinate frame $(x-y)$ and below the loading direction (when the cantilever is set to vibration).

practice, the LAZ is loaded along the cantilever's longitudinal axis. For instance, a writing direction along the cantilever corresponds to an axial loading of a LAZ as depicted in Fig. 5. For these two writing directions, along and transverse, we vary the nanograting orientation by changing the laser beam polarization and keeping the remaining exposure conditions constant - translation velocity of $5 \mathrm{~mm} / \mathrm{s}$, repetition rate of $750 \mathrm{kHz}$, and a pulse energy of $240 \mathrm{~nJ}$.

To model the in-plane transverse and longitudinal stiffness coefficients of a LAZ as a function of the nanograting orientation $(\theta)$, we first establish the stiffness matrix of a projected portion of the nanogratings in the local coordinate frame (1-2), as illustrated in Fig. 5. Second, to obtain the elastic properties in the global coordinate frame, we introduce a rotation of the local coordinate frame with respect to the global coordinate frame $(x-y)$. We treat the nanograting modification equivalently as a fiber composite and we assume that it is defining an orthotropic material [46]. The local coordinate frame $1-2$ is defined such that the 1 and 2 directions are along and transverse to the nanogratings, respectively. The in-plane stress-strain relationship for an orthotropic material is expressed by the matrix form in Eq. (5). With $Q_{l}$ as a symmetric $3 \times 3$ stiffness matrix, $Q_{l, 11}$ and $Q_{l, 22}$ are the stiffness elements along and transverse to the nanograting orientation, respectively, and $Q_{l, 12}$ and $Q_{l, 21}$, are the cross-coefficients. Finally, $Q_{1212}$ is equal to the shear modulus $\left(G_{l, 12}\right)$ :

$$
\left[\begin{array}{c}
\sigma_{11} \\
\sigma_{22} \\
\tau_{12}
\end{array}\right]=\underline{Q}_{l}\left[\begin{array}{l}
\epsilon_{11} \\
\epsilon_{22} \\
\gamma_{12}
\end{array}\right]=\left[\begin{array}{ccc}
Q_{l, 11} & Q_{l, 12} & 0 \\
Q_{l, 21} & Q_{l, 22} & 0 \\
0 & 0 & Q_{l, 1212}
\end{array}\right]\left[\begin{array}{c}
\epsilon_{11} \\
\epsilon_{22} \\
\gamma_{12}
\end{array}\right]=\frac{1}{1-v_{l, 12} v_{l, 21}}\left[\begin{array}{ccc}
E_{l, 11} & E_{l, 11} v_{21} & 0 \\
E_{l, 22} v_{12} & E_{l, 22} & 0 \\
0 & 0 & G_{l, 12}\left(1-v_{l, 12} v_{l, 21}\right)
\end{array}\right]\left[\begin{array}{c}
\epsilon_{11} \\
\epsilon_{22} \\
\gamma_{12}
\end{array}\right] .
$$




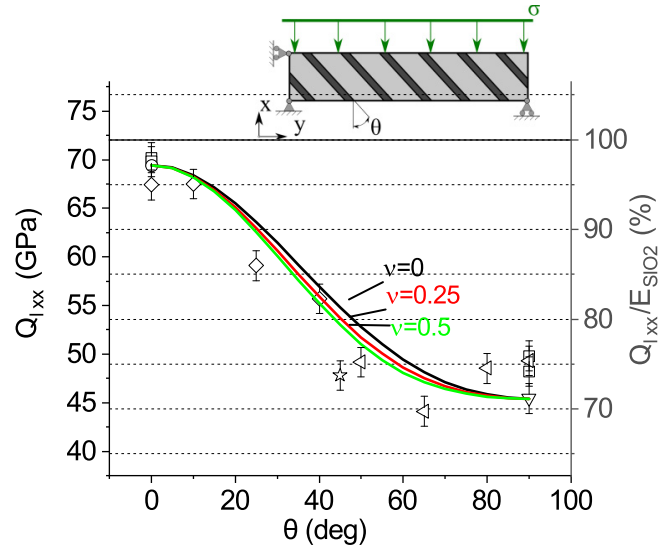

(a)Transverse Young's modulus $Q_{l, x x}$.

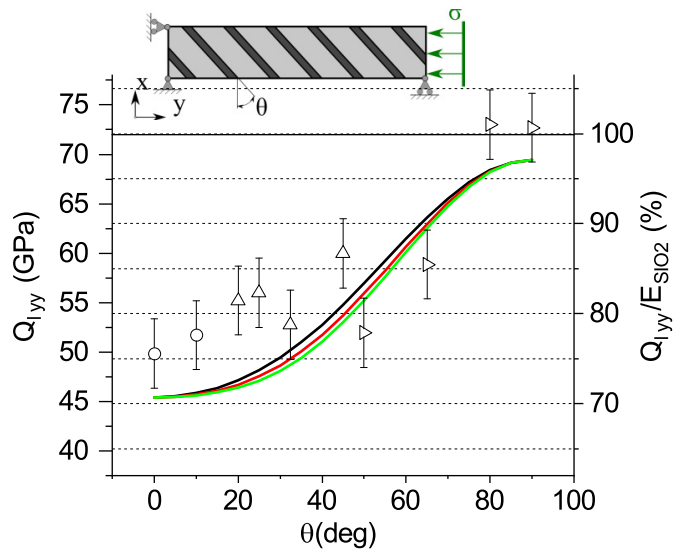

(b)Axial Young's modulus $Q_{l, y y}$.

FIG. 6. Estimation of the LAZ's Youngs modulus, $Q_{l, x x}$ and $Q_{l, y y}$, as a function of nanograting orientations $(\theta)$ defined with respect to the $x$ axis of the global coordinate frame. The experimental estimation is illustrated by a set of markers that correspond to a given specimen measured. The result of the modeling is shown with solid lines for different Poisson ratios $(v)$. The loading case is illustrated above each figure.

The elements of the matrix are expressed in terms of the material properties $E_{\text {. }}, v$. and $G_{l, 12}$, where $E_{l, 11}$ and $E_{l, 22}$ are the Young's modulus in the longitudinal and transverse directions. $v_{l, 12}$ and $v_{l, 21}$ are the Poisson's ratios and they can also be determined by the rule of mixtures, for instance, for the longitudinal direction, $v_{l, 12}=v_{\mathrm{nl}} V_{\mathrm{nl}}+v_{\mathrm{pl}} V_{\mathrm{pl}}$. For the Poisson's ratio in the transverse direction, we use the symmetry property of $Q_{l}$ and rewrite the diagonal elements, as $-v_{21}=$ $E_{l, 11} / E_{l, 22} v_{l, 12}$. We can further detail the coefficients $E_{l, 11}$ and $E_{l, 22}$, as we did with the discrimination experiment, in terms of the experimentally retrieved Young's moduli of the local layers, as $E_{l, 11}=E_{\mathrm{nl}} V_{\mathrm{nl}}+E_{\mathrm{pl}} V_{\mathrm{pl}}$ and $E_{l, 22}=E_{\mathrm{nl}} E_{\mathrm{pl}} / E_{\mathrm{nl}} V_{\mathrm{pl}}+$
$E_{\mathrm{pl}} V_{\mathrm{nl}} . G_{l, 12}$ is also expressed as a function of the local shear modulus, $G_{\mathrm{nl}}$ and $G_{\mathrm{pl}}$, and the volume fractions as $G_{l, 12}=G_{\mathrm{nl}} G_{\mathrm{pl}} / G_{\mathrm{nl}} V_{\mathrm{pl}}+G_{\mathrm{pl}} V_{\mathrm{nl}}$. Assuming that the layers are isotropic, we can express the local shear modulus in terms of the corresponding Young's modulus and Poisson's ratio, $G_{\mathrm{nl}}=E_{\mathrm{nl}} / 2\left(1+v_{\mathrm{nl}}\right)$ and $G_{\mathrm{pl}}=E_{\mathrm{pl}} / 2\left(1+v_{\mathrm{pl}}\right)$.

At this stage, we have expressed in-plane elastic properties as a function of the local material properties. In the next step, we introduce a rotation operator to model the nanograting orientation, by means of a coordinate transformation from the 1-2 (local) to $x-y$ (global) coordinate frame, see Eq. (6).

$$
\begin{gathered}
{\left[\begin{array}{c}
\sigma_{x x} \\
\sigma_{y y} \\
\tau_{x y}
\end{array}\right]=\underline{R}_{\sigma} \underline{Q}_{l} \underline{R}_{\sigma}^{T}\left[\begin{array}{c}
\epsilon_{x x} \\
\epsilon_{y y} \\
\gamma_{x y}
\end{array}\right]=\left[\begin{array}{ccc}
Q_{l, x x} & Q_{l, x y} & Q_{l, x x y} \\
Q_{l, y x} & Q_{l, y y} & Q_{l, y x y} \\
Q_{l, x x y} & Q_{l, y x y} & Q_{l, x y x y}
\end{array}\right]\left[\begin{array}{c}
\epsilon_{x x} \\
\epsilon_{y y} \\
\gamma_{x y}
\end{array}\right]} \\
\text { with; } \underline{R}_{\sigma}=\left[\begin{array}{ccc}
\cos ^{2} \theta & \sin ^{2} \theta & -2 \cos \theta \sin \theta \\
\sin ^{2} \theta & \cos ^{2} \theta & 2 \cos \theta \sin \theta \\
\cos \theta \sin \theta & -\cos \theta \sin \theta & \cos ^{2} \theta-\sin ^{2} \theta
\end{array}\right] .
\end{gathered}
$$

The elements $Q_{l, x x}$ and $Q_{l, y y}$ are of particular interest for us as these are the quantities we measure. These two terms are expressed in Eqs. (7) and (8) and can be expressed as function of the local Young's moduli, $E_{\mathrm{nl}}$ and $E_{\mathrm{pl}}$, the nanograting orientation, $\theta$, and the local Poisson's ratios, $v_{\mathrm{pl}}$ and $\nu_{\mathrm{nl}}$. As the Poisson's ratio is not known, we will evaluate $Q_{l, x x}$ and $Q_{l, y y}$ for a set of different values of $v_{\mathrm{pl}}$ and $v_{\mathrm{nl}}$ equal to the one of fused silica, $v_{\mathrm{SiO}_{2}}=0.17$,

$$
\begin{aligned}
& Q_{l, x x}=Q_{l, 11} \cos ^{4} \theta+\frac{1}{2}\left(Q_{l, 12}+2 Q_{l, 1212}\right) \sin ^{2} 2 \theta+\sin ^{4} \theta Q_{l, 22}, \\
& Q_{l, y y}=Q_{l, 22} \cos ^{4} \theta+\frac{1}{2}\left(Q_{l, 12}+2 Q_{l, 1212}\right) \sin ^{2} 2 \theta+\sin ^{4} \theta Q_{l, 11} .
\end{aligned}
$$

In one exposure, the lines are written alternating positive and negative writing directions, as illustrated in Fig. 5. The polarization for the writing directions is controlled such that the nanogratings are mirrored with respect to the axis along the cantilever, to avoid parasitic effects due the expansion in the transverse direction of the cantilever. The estimations of the model and experiments for both loading directions are depicted in Figs. 6(a) (transverse, $Q_{l, x x}$ ) and 6(b) (axial,
$\left.Q_{l, y y}\right)$, as a function of the nanograting orientation $(\theta)$ defined with respect to the $x$ axis of the global coordinate frame. The experimental estimation are illustrated by markers specific to a given measured specimen. The mathematical estimation is shown with a set of solid lines representing different Poisson's ratios $\left(v_{p l}\right)$. On top of both figures, the loading case for a given angle is illustrated. The experimental values follow the same trend as the model. Considering $Q_{l, x x}$, the stiffness decreases 
steeply from $0^{\circ}$ to $45^{\circ}$, as the model does. From $45^{\circ}$ and $70^{\circ}$, the experiment tends to deviate from the model, where the experiments show a dip. For experimental estimation of $Q_{l, y y}$, a similar trend is observed, but mirrored.

In the domain where the experiments deviate from the model, the cross product of Eqs. (7) and (8) is dominant and hence the shear modulus. A possible explanation is that the model overestimates the shear modulus $[47,48]$. Another possibility is that the nanogratings are not well developed for theses polarization angles. Zimmermann et al. [9] show that the nanograting orientation other than parallel or perpendicular to the writing direction requires a higher pulse number to fully develop. Remarkably, considering the inherent complexity of these experiments and the assumption made, there is a rather good agreement between our model and the experimental data, suggesting the validity of this modeling approach and the estimation of the local properties in the previous section.

As a final remark, an interesting additional tuning parameter could be the periodicity of the porous layers, as an increase of periodicity could potentially result in a higher density of porous layers and, hence, a further contrast increase of the Young's modulus in the orthogonal direction and, consequently, a higher degree of anisotropy.

\section{CONCLUSION}

In this paper, we reported on the characterization of local and in-plane elastic properties of femtosecond laser-induced nanogratings in bulk fused silica. The estimation of the values for individual layers is in good agreement with earlier observations of the local refractive index changes. This further confirms that the regions between porous layers, found in the nanograting modification, has similar characteristics as a regime-I modification - a denser structure, with a higher Young's modulus with respect to the pristine material. Interestingly, the estimated elastic properties of this region are significantly higher compared to a regime-I modification. This observation suggests that the densification mechanism of this layer is more complex than the regime-I modification. Based on the local properties estimation, a mathematical description of the in-plane elastic properties is derived and is experimentally validated for axial and transverse properties. This result shows that we can tailor the Young's modulus depending on the nanograting orientation. Finally, this study demonstrates "laser direct-write nanocomposites" obtained without adding another material, but rather by reorganizing the distribution of matter from the inside, in a controllable way and according an arbitrary pattern. These "direct-write" nanocomposites are particularly attractive for novel types of precision resonators and mechanical microdevices with anisotropic and tuned elastic properties.

\section{ACKNOWLEDGMENT}

The Galatea Lab acknowledges the sponsorship of Richemont International SA.
[1] C. B. Schaffer, A. Brodeur, J. F. García, and E. Mazur, Meas. Sci. Technol. 12, 1784 (2001).

[2] Y. Shimotsuma, P. G. Kazansky, J. Qiu, and K. Hirao, Phys. Rev. Lett. 91, 247405 (2003).

[3] S. Richter, A. Plech, M. Steinert, M. Heinrich, S. Döring, F. Zimmermann, U. Peschel, E. B. Kley, A. Tünnermann, and S. Nolte, Laser Photon. Rev. 6, 787 (2012)

[4] A. Champion and Y. Bellouard, Opt. Mater. Express 2, 789 (2012).

[5] M. Lancry, B. Poumellec, J. Canning, K. Cook, J.-C. Poulin, and F. Brisset, Laser Photon. Rev. 7, 953 (2013).

[6] W. Yang, E. Bricchi, P. G. Kazansky, J. Bovatsek, and A. Y. Arai, Opt. Express 14, 10117 (2006).

[7] C. Hnatovsky, R. S. Taylor, P. P. Rajeev, E. Simova, V. R. Bhardwaj, D. M. Rayner, and P. B. Corkum, Appl. Phys. Lett. 87, 014104 (2005).

[8] S. Richter, M. Heinrich, S. Döring, A. Tünnermann, and S. Nolte, Appl. Phys. A 104, 503 (2011).

[9] F. Zimmermann, A. Plech, S. Richter, A. Tünnermann, and S. Nolte, Laser Photon. Rev. 10, 327 (2016).

[10] S. Richter, F. Jia, M. Heinrich, S. Döring, U. Peschel, A. Tünnermann, and S. Nolte, Opt. Lett. 37, 482 (2012).

[11] Y. Liao, J. Ni, L. Qiao, M. Huang, Y. Bellouard, K. Sugioka, and Y. Cheng, Optica 2, 329 (2015).

[12] V. R. Bhardwaj, E. Simova, P. P. Rajeev, C. Hnatovsky, R. S. Taylor, D. M. Rayner, and P. B. Corkum, Phys. Rev. Lett. 96, 057404 (2006).
[13] M. Beresna, M. Gecevičius, P. G. Kazansky, T. Taylor, and A. V. Kavokin, Appl. Phys. Lett. 101, 053120 (2012).

[14] M. Beresna, M. Gecevičius, P. G. Kazansky, and T. Gertus, Appl. Phys. Lett. 98, 201101 (2011).

[15] J. Zhang, M. Gecevičius, M. Beresna, and P. G. Kazansky, Phys. Rev. Lett. 112, 033901 (2014).

[16] B. McMillen and Y. Bellouard, Opt. Express 23, 86 (2015).

[17] B. McMillen, C. Athanasiou, and Y. Bellouard, Opt. Express 24, 27239 (2016).

[18] C.-E. Athanasiou and Y. Bellouard, Micromachines 6, 1365 (2015).

[19] Y. Bellouard, Opt. Express 23, 29258 (2015).

[20] S. I. Nazir and Y. Bellouard, Micromachines (Basel) 10, 611 (2019).

[21] P. Vlugter, E. Block, and Y. Bellouard, Phys. Rev. Mater. 3, 053802 (2019).

[22] C. Corbari, A. Champion, M. Gecevičius, M. Beresna, Y. Bellouard, and P. G. Kazansky, Opt. Express 21, 3946 (2013).

[23] Y. Bellouard, in Femtosecond Phenomena and Nonlinear Optics III, Vol. 6400, edited by R. S. Sean and M. Kirkpatrick (SPIE, Bellingham, Washington, 2006).

[24] L. P. R. Ramirez, M. Heinrich, S. Richter, F. Dreisow, R. Keil, A. V. Korovin, U. Peschel, S. Nolte, and A. Tünnermann, Appl. Phys. A: Mater. Sci. Process. 100, 1 (2010).

[25] E. Bricchi, B. G. Klappauf, and P. G. Kazansky, Opt. Lett. 29, 119 (2004).

[26] J. M. Rimsza and J. Du, J. Am. Ceram. Soc. 97, 772 (2014). 
[27] T. Adachi and S. Sakka, J. Mater. Sci. 25, 4732 (1990).

[28] Y. Bellouard, A. Champion, B. McMillen, S. Mukherjee, R. R. Thomson, C. Pépin, P. Gillet, and Y. Cheng, Optica 3, 1285 (2016).

[29] K. Mishchik, C. D’Amico, P. K. Velpula, C. Mauclair, A. Boukenter, Y. Ouerdane, and R. Stoian, J. Appl. Phys. 114, 133502 (2013).

[30] Y. Bellouard, T. Colomb, C. Depeursinge, M. Dugan, A. A. Said, and P. Bado, Opt. Express 14, 8360 (2006).

[31] E. Bricchi and P. G. Kazansky, Appl. Phys. Lett. 88, 111119 (2006).

[32] K. M. Davis, K. Miura, N. Sugimoto, and K. Hirao, Opt. Lett. 21, 1729 (1996).

[33] K. Mishchik, G. Cheng, G. Huo, I. M. Burakov, C. Mauclair, A. Mermillod-Blondin, A. Rosenfeld, Y. Ouerdane, A. Boukenter, O. Parriaux, and R. Stoian, Opt. Express 14, 3785 (2006).

[34] J. Hernandez-Rueda, J. Clarijs, D. van Oosten, and D. M. Krol, Appl. Phys. Lett. 110, 161109 (2017).

[35] R. Bruckner, J. Non-Cryst. Solids 5, 123 (1970).

[36] W. Primak, J. Appl. Phys. 43, 2745 (1972).

[37] A. Saliminia, N. T. Nguyen, S. L. Chin, and R. Vallée, J. Appl. Phys. 99, 093104 (2006).
[38] T. Deschamps, J. Margueritat, C. Martinet, A. Mermet, and B. Champagnon, Sci. Rep. 4, 7193 (2014).

[39] M. Guerette, M. R. Ackerson, J. Thomas, F. Yuan, E. Bruce Watson, D. Walker, and L. Huang, Sci. Rep. 5, 15343 (2015).

[40] R. Le Parc, C. Levelut, J. Pelous, V. Martinez, and B. Champagnon, J. Phys.: Condens. Matter 18, 7507 (2006).

[41] H. Kakiuchida, K. Saito, and A. J. Ikushima, Jpn. J. Appl. Phys. 43, L743 (2004).

[42] U. Haken, O. Humbach, S. Ortner, and H. Fabian, J. Non-Cryst. Solids 265, 9 (2000).

[43] M. Lancry, B. Poumellec, A. Chahid-Erraji, M. Beresna, and P. G. Kazansky, Opt. Mater. Express 1, 711 (2011).

[44] C. Z. Tan, J. Arndt, and H. S. Xie, Phys. B 252, 28 (1998).

[45] A. Champion, M. Beresna, P. Kazansky, and Y. Bellouard, Opt. Express 21, 24942 (2013).

[46] W. C. Young and R. G. Budynas, Roark's Formulas for Stress and Strain, 7th ed. (McGraw-Hill, New York, USA, 2002).

[47] O. Pierard, C. Friebel, and I. Doghri, Compos. Sci. Technol. 64, 1587 (2004).

[48] G.-Z. Kang and Q. Gao, Elsevier Composites: Part A 33, 657 (2002). 